\title{
Patterning of antibodies using flexographic printing
}

Christopher O. Phillips ${ }^{2}$, Sridhar Govindarajan ${ }^{2}$, Simon M. Hamblyn ${ }^{1}$, R. Steven Conlan ${ }^{2}$, David T. Gethin $^{1}$, Tim C. Claypole ${ }^{l}$

1. Welsh Centre for Printing and Coating, College of Engineering, Swansea University, Singleton Park, Swansea, SA2 8PP, UK.

2. Institute of Life Sciences, College of Medicine, Swansea University, Singleton Park, Swansea, SA2

$$
\text { 8PP, UK. }
$$

c.o.phillips@swansea.ac.uk

Abstract

Antibodies were patterned onto flexible plastic films using the flexographic printing process. An ink formulation was developed using high molecular weight polyvinyl alcohol in carbonate-bicarbonate buffer. In order to aid both antibody adhesion and quality of definition in the printed features, a nitrocellulose coating was developed; this was capable of being discretely patterned thus increasing the signal to noise ratio of an antibody array. Printing antibody features such as dots, squares, text and fine lines were reproduced effectively. Furthermore, this process could be easily adapted for printing of other biological materials, including but not limited to, enzymes, DNA, proteins, aptamers and cells.

\section{Introduction}

Antibodies and proteins have been deposited onto various surfaces, for use in biological or chemical assays, using methods such as inkjet [1-5], micro-contact printing [6,7], capillary drop patterning [8] or other drop-on-demand techniques such as BIODOT [9]. A range of surfaces have been printed on such 
as glass slides, nitrocellulose coated slides, plastics, papers (where biological materials, are imbedded for detection via by colorimetric analysis; so-called bioactive papers $[4,10])$ or membranes for lateral flow immunochromatographic methods [5]. The ability to deposit arrays of antibodies using reel-to-reel printing processes such as flexography would greatly increase the speed of production when compared with these current patterning process. Faster patterning methods could then be exploited for applications such as enzyme linked immunoassay (ELISA) and biosensors in order to reduce testing time, equipment requirements and expertise. The availability of low cost, disposable assay technology will aid the early diagnosis of disease and facilitate cost effective health management.

Patterning proteins with piezoelectric inkjet, for example, requires a very narrow operational window in terms of rheological properties and suitable additives, is unpredictable, with a tendency to clog and has a very slow print speed $[1,11]$. Spot or line deposition using systems such as BIODOT RR4510 reel-to-reel dispensing platform [12] can produce speeds of up to $0.1 \mathrm{~m} / \mathrm{s}$ but can only process narrow width membranes (less than $100 \mathrm{~mm}$ ) with very limited complexity in deposition patterns. Commercial wide format inkjet printers are much larger and have much greater speeds than laboratory equipment. For example, a wide format inkjet printer produced by Roland DG (VersaArt ${ }^{\mathrm{TM}} \mathrm{RS}-640$ [13]) can print on a 1.6 meter reel giving a maximum coverage of $21 \mathrm{~m}^{2} /$ hour, depending on print resolution requirements. However, larger flexographic printings presses can run at hundreds of meters per minute [14]; making them substantially faster than any current inkjet process.

Flexographic printing is a high speed reel-to-reel process $[14,15]$ traditionally used for graphics printing in applications such as packaging. The process is continuous and usually uses a reel of substrate, but can use sheets. Applications of flexographic printing for functional materials have so far been focused on electronics applications [16] and have not extended to biological applications. By printing onto a plastic film substrate, an array of antibodies can be produced to act as a printed ELISA which is subjected to the washing and incubation stages that would normally take place in a microwell 
plate [1]. This article therefore details the development of new systems for patterning of antibodies onto low cost commercial polymer films using flexographic printing. Flexography uses a photopolymer relief plate as an image carrier with a raised printing surface onto which ink is transferred using an engraved cylinder known as an anilox. The ink is then transferred from the plate to a substrate such as paper or plastic film (figure 1). The frequency and size of cells engraved in the anilox determines the volume of ink that can be held in the cells and is the main controlling factor in the amount of ink transferred. The ink capacity of the anilox cylinder is normally quoted in cubic centimeters of ink per meter squared of anilox surface and is an approximate indicator of the maximum theoretical ink transfer to the substrate.

Figure 1. Schematic of the flexographic printing process.

In order to deliver antibodies by printing, a suitable ink has to be developed and an appropriate carrier film substrate selected. An antibody "ink" formulation has two purposes; firstly to act as a vehicle to apply the antibody to a substrate and secondly to allow the antibody to survive the stresses imposed by printing (due to shearing, film splitting etc.) and drying, which causes changes in ionic strength, antibody hydration, $\mathrm{pH}$ and surface energy all of which can affect the protein structure and reduce bioactivity [17], while permitting antibody binding to the surface. However, the requirements for antibody binding and functionality must be balanced against the requirements for successful printing; antibody inks have to be water based and are thus difficult materials to use with plastic films as they struggle to adequately wet the surface. Surfactants are useful in reducing the surface tension of waterbased systems to facilitate the transfer of ink through the various stages of the printing process as well as the subsequent wetting of the liquid onto a surface. In addition, flexography typically requires a viscosity range of approximately 0.05 to $0.5 \mathrm{~Pa} \mathrm{~s}$ [14] which necessitates the addition of polymeric binders. Antibody activity must not be compromised by the additives, which must also provide the appropriate rheological properties for the printing process; both in terms of viscosity and surface 
tension. However, excessive amounts of polymer are not desirable as they may impair diffusion of the antibodies to the surface and analyte binding to the antibody during testing $[11,18]$. The additives can have a beneficial effect if selected appropriately. Hydroxylated polymers were used as they have been reported to improve the functionality of antibodies printed onto microarray slides [7] and are soluble in water. A variety of hypotheses explaining the mechanisms of how these additives protect are discussed by $\mathrm{Wu}$ and Grainger [17], who found polyvinyl alcohol (PVA) (9000-10,000 molecular weight) to be the most useful additive in facilitating even distribution, good spot morphology and preservation of antibody bioactivity, with reasonable activity preserved after storage for 1 month at $4^{\circ} \mathrm{C}$. Surfactants can also improve protein stability under high-shear processes and those which induce interfacial stress (such as printing) by displacing protein from the air-water interface [18, 19]. However, because surfactants migrate to interfaces they will interfere with adsorption of antibodies [20,21] and will also increase drop spreading. A range of polymers and surfactants were therefore tested both for their capabilities as rheology modifiers, to facilitate printing, and their effect on antibody activity and binding.

Carrier film selection is based on both the ability of the film to receive the ink during printing, allowing good print definition, and the ability of an antibody to bind to the surface. Commercial polymer films are fabricated from a range of different polymers and with different surface coatings. The functional groups on the surface of the polymer film might be exploitable for binding proteins. For example, acrylic contains the carbonyl group which could bind with the amino groups on the protein. Nitrocellulose is used in products specifically designed for adhesion of protein such as membranes for lateral flow and Western blots. The nitro groups on the nitrocellulose interact with the carbonyl groups in the protein and there is further interaction between the hydrophobic parts of the protein and the nitrocellulose [20-24]. Therefore, a range of films were tested for their ability to bind antibodies and a nitrocellulose-based coating was developed specifically for this purpose. 
Prior to printing antibodies, the appropriateness of various additives for the printing process was determined by evaluating their rheological characteristics. The effects of these additives on the efficacy of antibodies, as well as the effects of drying and shearing, were evaluated by measuring the fluorescein quenching activity of anti-FITC antibody. Antibody binding for different additive and surface combinations was then evaluated by measuring the presence of peroxidase labeled antibody on the surface. Once a suitable formulation was established, preliminary tests were carried out to establish the optimum print settings using a dye in place of antibodies. Antibodies were then printed using the optimum formulation and print settings and assessed by incubating with the secondary peroxidase labeled antibody to bind to the printed antibody. Finally, the ability of nitrocellulose coating to be discretely patterned onto a surface, to reduce non-selective binding, was explored.

\section{Experimental section}

Materials. The materials used in the experiments are as follows: Anti-FITC polyclonal serum produced in sheep (Ig-Innovations, Llandysul, UK), Anti-Sheep IgG (whole molecule)-Peroxidase antibody produced in donkey (Sigma Aldrich A3415), fluorescein sodium salt (Sigma Aldrich F6377) and a chemiluminescent substrate solution (Immobilon Western Chemiluminescent HRP Substrate, Millipore, WBKLS0100) were purchased or donated. PBS buffer (0.01 M phosphate buffer, $0.0027 \mathrm{M}$ potassium chloride and $0.137 \mathrm{M}$ sodium chloride, $\mathrm{pH} 7.4$, at $25^{\circ} \mathrm{C}$, Sigma Aldrich P4417), Carbonate buffer (0.05 M carbonate-bicarbonate buffer, $\mathrm{pH} 9.6$ at $25{ }^{\circ} \mathrm{C}$, Sigma Aldrich C3041) and blocking buffer (Sigma Aldrich B6429) were purchased and used as directed. Low molecular weight PVA (Poly(vinyl alcohol) Mw 9000 to 10,000 Sigma Aldrich 360627), high molecular weight PVA (Poly(vinyl alcohol) average Mw 130,000 Sigma Aldrich 563900), hydroxypropyl cellulose (Mw 1,000,000, Sigma Aldrich 191906), xanthan gum (Sigma Aldrich G1253), Triton X-100 (Sigma Aldrich X100) and Tween 20 (Sigma Aldrich T2700) were purchased and dissolved in double distilled water or buffer as required. Acrylic coated polypropylene film (Rayoface ${ }^{\mathrm{TM}}$ WPA59) were donated by Innovia Films Ltd, Cumbria, UK. Highly nitrated E-grade nitrocellulose (RS 120, Nobel NC Co Ltd. Bangkok, 
Thailand) and low nitration A-grade nitrocellulose (A500, Dow Wolff Cellulosics) were used to develop custom film coatings. Solvents including n-butyl acetate (Fisher Scientific B/4951/15) and propan-2-ol (Fisher Scientific P/7490/17) were used to dissolve the nitrocellulose.

\section{Methods and Equipment}

Antibody ink rheology. The viscosity of antibody ink formulations were measured using a rheometer (Gemini Bohlin Nano, Malvern Instruments) with a $2^{\circ} 55 \mathrm{~mm}$ stainless steel cone and a parallel plate held at $25^{\circ} \mathrm{C}$ over a shear rate range of 1 to $100 \mathrm{~s}^{-1}$. Surface tension was evaluated using the pendant drop method with a Fibrodat DAT1100 (Fibro System AB, Sweden).

Antibody efficacy in ink formulations and the effect of shear and drying. Antibody efficacy in the liquid phase was evaluated by monitoring the ability of anti-FITC to quench the fluorescence of fluorescein sodium salt in a 96-well plate (Microplate Microfluor 1 96-well flat bottom polystyrene solid black). Fluorescence measurements were carried out using a fluorescence plate reader (POLARstar Omega, BMG Labtech). An excitation wavelength of $485 \mathrm{~nm}$ was used with a measurement wavelength of $520 \mathrm{~nm}$. To evaluate the effect of shear on antibodies, $10 \%$ volume antiFITC serum in PBS was sheared at a rate of $25,000 \mathrm{~s}^{-1}$ for 30 minutes using a rheometer with a parallel plate. Liquid phase tests were performed at $0.025 \%$ antiserum concentration, with $10 \mathrm{nM}$ fluorescein in a $200 \mu \mathrm{L}$ well volume. To evaluate the effect of drying on antibodies, plate tests were performed to compare the efficacy of dried and then rehydrated antibodies compared with antibodies in the liquid phase. These tests used $20 \mu \mathrm{L}$ quantities of $0.25 \%$ antiserum pipetted into the wells and dried for 3 hours at $37^{\circ} \mathrm{C}$. After drying, $200 \mu \mathrm{L}$ of $10 \mathrm{nM}$ fluorescein was added and the fluorescence measured. In all cases, control samples were stored alongside test samples to prevent variations due to thermal history. 
Surface immobilization of antibodies. Surface immobilization of antibodies was compared for different formulation/surface combinations via the following procedure: labeled antibody (Anti-Sheep IgG-Peroxidase) at $0.25 \%$ was added to the formulation, applied to the surface in $3 \mu \mathrm{L}$ droplets via pipette and allowed to dry at $37^{\circ} \mathrm{C}$. The dried antibody deposit was washed three times in PBS-Tween or blocking buffer and then visualized by spraying chemiluminescent substrate solution which was activated by the peroxidase enzyme. The chemiluminescence imager was a ChemiDoc XRS $+\mathrm{System}$ from Bio-Rad.

A range of hydroxylated polymers and surfactants were tested as well as both PBS and coating buffers as listed in the materials section. A range of polymer film substrates were tested as well as a custom nitrocellulose coating. Surface modification using ultraviolet light was also investigated. Nitrocellulose is available in a range of grades depending on the nitrogen content. However, the degree of nitration determines which solvents can be used to dissolve and dilute the resin, with higher nitrogen content requiring more aggressive solvents. To make a formulation suitable for bar coating, highly nitrated Egrade nitrocellulose was dissolved in n-butyl acetate then further diluted using propan-2-ol (1 part nitrocellulose, 3 parts acetate, 2 parts propanol by weight) to give appropriate viscosity. This coating was applied to plastic film using an automated bar coater (K Control Coater, RK PrintCoat Instruments Ltd., Herts, UK) to give a thin layer of dry nitrocellulose. Using a bar wound with 0.0015 inch $(0.0381$ $\mathrm{mm}$ ) wire, a dry film thickness of approximately $0.6 \mu \mathrm{m}$ was obtained.

In order to further investigate the ability of the nitrocellulose coating to adhere antibodies, E-grade nitrocellulose solution was pipetted into the wells of a 96-well plate; $20 \mu \mathrm{L}$ quantities covered the base of the well (3\% in n-butyl acetate and propan-2-ol to enable pipetting). After drying, $20 \mu \mathrm{L}$ of $5 \%$ antiFITC in $2.5 \%$ high molecular weight PVA in carbonate buffer were pipetted into both nitrocellulose coated and normal uncoated wells of the same plate and allowed to dry at $37^{\circ} \mathrm{C}$ for three hours. The plate was washed three times in PBS-Tween. During the first wash, the wells were immersed for 5 
minutes and for the second and third washes, a longer period of 45 minutes was used. $200 \mu \mathrm{L}$ of $10 \mathrm{nM}$ fluorescein was added and the fluorescence quenching by anti-FITC measured. Separate fluorescein standards were used for coated and uncoated wells.

Printing methods. Having established the optimum formulation/substrate combination (Table 1), laboratory flexographic printing tests were carried out using an IGT F1 Printability tester (IGT Testing Systems, Amsterdam, NL), a small scale flexographic printer. A range of parameters can be altered including print speed ( 0.2 to $1.5 \mathrm{~m} / \mathrm{s})$, pressure between anilox and plate cylinders and between plate and impression cylinders $(25$ to $500 \mathrm{~N})$, anilox volume $\left(8,12\right.$ and $\left.24 \mathrm{~mL} / \mathrm{m}^{2}\right)$ and anilox revolutions against the plate prior to printing (pre-inking 0 to 20 revolutions). The photopolymer plates were 1.7 mm thickness AFP (D)SH (Asahi Photoproducts (UK) Ltd.). Each printing cycle uses approximately 0.5 to $1 \mathrm{~mL}$ of ink and produces a print of $50 \mathrm{~mm}$ width and approximately $600 \mathrm{~mm}$ in length. The wastage of ink depends on the anilox (i.e. film thickness transferred) and the proportion of the plate area that is printed. The low volume requirements makes it convenient for testing small batches of expensive formulations which would be required in hundreds of $\mathrm{mL}$ quantities if a full-scale press was used. Prints were produced using a range of printing parameters with dye in place of antibodies in order to select the optimum settings for antibody printing.

The resulting optimum print settings (Table 2) were then used to print antibody ink (Table 1) using $2 \%$ anti-FITC. After printing, samples were placed in a refrigerator for later visualization. To measure the activity of the printed antibodies, the anti-sheep peroxidase labeled antibody was attached to the printed anti-FITC and visualized using the following steps: the printed antibody samples were washed three times with blocking buffer to remove any unattached protein $(3 \times 5$ minute washes at $75 \mathrm{rpm}$ on a shaker). The printed samples were then incubated in a solution of $0.1 \% \mathrm{vol}$ anti-sheep peroxidase labeled antibody in blocking buffer to bind to the printed anti-FITC under gentle agitation for a period 
of one hour. This was washed in blocking buffer and visualized under the chemiluminescence imager by spraying the chemiluminescent substrate on the sample.

Nitrocellulose patterning. The ability of the nitrocellulose coating to be patterned was then tested in order to reduce non-specific binding. The solid content of the E-grade nitrocellulose coating was reduced to around $8 \%$ to give an appropriate print viscosity. A less nitrated A-grade formulation (10\% nitrocellulose, 20\% n-butyl acetate and 70\% propan-2-ol) was also used, although this has fewer nitro groups for binding. Printing was carried out using the IGT F1 $(8 \mathrm{~mL} / \mathrm{m} 2$ anilox, $0.2 \mathrm{~m} / \mathrm{s}$ print speed, 75 $\mathrm{N}$ anilox pressure, $150 \mathrm{~N}$ printing pressure, 4 anilox revolutions). In addition, an IGT G1 Printability tester (IGT Testing Systems, Amsterdam, NL), a simulator of the gravure process was used with an intermediate printing pressure of $500 \mathrm{~N}$ and an intermediate speed setting of 3 out of 5 . Dye was added to visualize the print, and a visual assessment was made.

The findings of these experiments are presented in the following sections. For brevity, only selected data is shown.

\section{Results}

Rheological characteristics of additives. Low molecular weight polymers have a smaller influence on rheology than larger polymers and thus are needed in greater concentrations in order to facilitate printing. Low molecular weight PVA produced a viscosity of $0.01 \mathrm{~Pa}$ s at approximately $9 \%$ by mass concentration. This enabled printing but gives an excessive amount of polymer in the print. By contrast, high molecular weight PVA gave this viscosity at $2.5 \%$ concentration (neither varied with shear rate). Low molecular weight PVA exhibited a noticeable surfactant effect $(0.5 \%$ by mass reduced the surface tension of water from 73 to $44 \mathrm{mN} / \mathrm{m}$ ) while high molecular weight PVA reduced surface tension only slightly from 73 to $66 \mathrm{mN} / \mathrm{m}$. High molecular weight cellulose based polymers were found to confer good rheological properties, with only small concentrations of polymer required to enable 
printing (less than $1 \%$ for hydroxypropyl cellulose (Mw 1,000,000) and xanthan gum) as well as being shear thinning; a desirable quality for most forms of volume printing. However, they were found to foam upon mixing and printing; giving poor print quality. Dilutions of 0.05 and 0.5 vol \% Tween 20 were found to reduce the surface tension of water to 44.4 and 39.9 dynes/cm respectively. Triton X-100 was yet more effective, with dilutions of 0.01 and 0.05 vol \% giving surface tensions of 39.4 and 31.4 dynes/cm respectively. Further increases in concentration did not reduce the surface tension any further. When used in conjunction with low molecular weight PVA, further reductions in surface tension were observed. These additives can aid printing, but this has to be balanced against their effect on antibody functionality and binding.

Antibody activity in liquid phase. The hydroxylated polymers tested were not found to reduce the fluorescence quenching activity of anti-FITC in the liquid phase and are therefore suitable candidate materials for antibody printing. Similarly, there was no reduction observed with the non-ionic surfactants (Triton X-100 and Tween 20) at the concentrations required for effective surface tension reduction. However, ionic surfactants (Sodium dodecyl sulphate and Cetyl trimethylammonium bromide) were found to reduce the fluorescence quenching ability of anti-FITC. The efficacy of the antiserum did not appear to change as a result of it being dried in PBS. This is demonstrated in figure 2, in which the amount of fluorescence quenching, expressed as a percentage loss in fluorescence compared with the standard, is shown for liquid phase antiserum and three separate tests on dried and rehydrated antiserum. For antibodies sheared at $25,000 \mathrm{~s}^{-1}$ for 30 minutes, there was no discernible difference between the fluorescence quenching levels of sheared and non-sheared antiserum, suggesting that there was no damage due to shear as demonstrated in figure 3.

Figure 2. Fluorescence quenching for anti-FITC in PBS compared with rehydrated anti-FITC dried in PBS. 
Figure 3. Fluorescence quenching for unsheared anti-FITC and anti-FITC sheared at $25,000 \mathrm{~s}^{-1}$ for 30 minutes (0.025\% antiserum with $10 \mathrm{nM}$ Fluorescein (error bars show standard deviation)).

Surface immobilization of antibodies. The range of additives differed in their ability to allow antibodies to bind to surfaces (observed by a variation in chemiluminescence intensity). Low molecular weight forms of polymers such as PVA and polyethylene glycol were found to hinder adhesion of antibodies to the surface when compared with antibodies in buffer only. The polymers readily washed away due to their high solubility, taking much of the antibodies with them. Improvements were observed with hydroxypropyl cellulose and xanthan gum but the best performance was observed for high molecular weight PVA (which performed similarly to antibody-buffer solution at $2.5 \%$ concentration). Addition of surfactant (Triton X-100) into the antibody solution gave increased spreading of deposited droplets, but reduced antibody adsorption for a given surface area due presumably to a reduction in hydrophobic interactions between the antibody and the substrate. In these tests, the alkaline carbonate buffer gave greater immobilization to the surfaces than a neutral PBS buffer.

A greater amount of antibody immobilization was observed from a nitrocellulose coated film than any of a range of polymer films without this applied layer. Immobilization on an acrylic surface from a commercial film (WPA59) is compared with the nitrocellulose coating in figure 4 (this test used 2.5\% high molecular weight PVA in coating buffer). The presence of the peroxidase tagged antibody is denoted by the color intensity of the droplet, with a darker color signifying greater presence of peroxidase, and hence antibody, on the nitrocellulose coated sample. The average intensity of the chemiluminescent signal for the droplets was approximately 1.6 times higher on the nitrocellulose coating; 43817 (9552 standard deviation) compared with 26906 (6246 standard deviation) on the acrylic surface. Background intensities of the nitrocellulose and acrylic surfaces were 6835 and 2813 respectively. Discrete patterning of the nitrocellulose coating would therefore increase the signal to 
noise ratio. The fluorescence quenching of anti-FITC (in 2.5\% high molecular weight PVA and carbonate buffer) dried in and then washed from normal and nitrocellulose coated wells of a 96-well plate is compared in figure 5. There was a substantial improvement in fluorescence quenching in the nitrocellulose coated wells when compared with untreated wells, with quenching increasing over time. This test further confirms that the antibodies are active upon drying and rehydration in an ink formulation.

Figure 4. Anti-Sheep IgG (whole molecule)-Peroxidase, visualized by chemiluminescence, on acrylic (left) and nitrocellulose coated surfaces (right). Average intensity of the chemiluminescent signal for the droplets was 1.6 times higher on the nitrocellulose coating than the acrylic surface. Formulation contains $0.25 \%$ antibody and $2.5 \%$ high molecular weight PVA in carbonate buffer.

Figure 5. Comparison of dried then washed antibody deposit on normal polystyrene plate surface and nitrocellulose coated surface of a 96 -well plate. Formulation contains $2 \%$ anti-FITC serum and $2.5 \%$ high molecular weight PVA in carbonate buffer.

Exposure of the substrate to ultraviolet radiation gave a similar outcome to adding surfactants i.e. improved the wetting characteristics with increased droplet spreading, but reduced antibody adsorption.

The series of tests performed previously suggested that the optimum combination of ink formulation and surface was $2.5 \%$ high molecular weight PVA in carbonate buffer, no additional surfactant and depositing on to a nitrocellulose surface. This combination was therefore used to optimize the printing process and is summarized in Table 1.

Table 1. Optimum ink formulation and substrate for antibody binding

\section{Parameter $\quad$ Setting}




\begin{tabular}{|l|l|}
\hline Polymer & $\begin{array}{l}\text { 2.5\% by mass Poly(vinyl alcohol) } \\
\text { (average Mw 130,000, Sigma Aldrich } \\
563900)\end{array}$ \\
\hline Surfactant & None \\
\hline Buffer & $\begin{array}{l}\text { 0.05 M carbonate-bicarbonate buffer, } \\
\text { pH 9.6, Sigma Aldrich C3041) }\end{array}$ \\
\hline Surface & E-grade nitrocellulose coating \\
\hline
\end{tabular}

Process optimization by printing with dye in place of antibodies. At the lowest tested anilox volume $\left(8 \mathrm{~mL} / \mathrm{m}^{2}\right)$, the choice of substrate coating or speed made only a small difference to print quality. However, as the anilox volume was increased (to 12 and $24 \mathrm{~mL} / \mathrm{m}^{2}$ ), the print quality was substantially reduced on the uncoated substrate due to dewetting of the ink formulation. This was mitigated by using an increased print speed which reduced the time available for dewetting of the antibody ink. The nitrocellulose coated film reduced the sensitivity of print quality to the printing parameters and gave substantially better printed images than uncoated films, especially at high anilox volumes and low printing speeds. This is highlighted in figure 6 , which compares printed images on nitrocellulose and acrylic surfaces at the optimum settings $\left(8 \mathrm{~mL} / \mathrm{m}^{2}\right.$ anilox and a print speed of $\left.1 \mathrm{~m} / \mathrm{s}\right)$ and the worst settings $\left(24 \mathrm{~mL} / \mathrm{m}^{2}\right.$ anilox and a print speed of $\left.0.2 \mathrm{~m} / \mathrm{s}\right)$. As the coating was more receptive to the ink, it removed the need for the addition of surfactants as a means of improving print quality and preventing dewetting. Both $\mathrm{E}$ and A grade nitrocellulose coatings permitted good print quality for the antibody ink formulation. Low pressures gave the best results, with high pressures causing distortion of the printed features due to compression of the printing plate. The optimized parameters for antibody printing are listed in Table 2.

Figure 6. Printed dye on nitrocellulose (left) and acrylic (right) using $8 \mathrm{~mL} / \mathrm{m}^{2}$ anilox and $1 \mathrm{~m} / \mathrm{s}$ print speed (top) and $24 \mathrm{~mL} / \mathrm{m}^{2}$ anilox and $0.2 \mathrm{~m} / \mathrm{s}$ print speed (bottom) - ink uses $2.5 \%$ high molecular weight PVA in carbonate buffer.

Table 2. Optimized printing parameters used in antibody printing 


\begin{tabular}{|l|l|}
\hline Parameter & Setting \\
\hline Anilox volume & $8 \mathrm{~mL} / \mathrm{m}^{2}$ \\
\hline Print speed & $1 \mathrm{~m} / \mathrm{s}$ \\
\hline Anilox pressure & $75 \mathrm{~N}$ \\
\hline Print pressure & $150 \mathrm{~N}$ \\
\hline $\begin{array}{l}\text { Number of anilox } \\
\text { revolutions }\end{array}$ & 4 \\
\hline Substrate & $\begin{array}{l}\text { Nitrocellulose coated } \\
\text { WPA59 }\end{array}$ \\
\hline
\end{tabular}

Printing of antibodies. Chemiluminescence images of the visualized printed antibodies are shown in figure 7; from an exposure time of 10 seconds using the optimized formulation (Table 1) and printing settings (Table 2) discussed previously. The images confirm that the printing methods and formulation were appropriate to obtain sufficient detail and antibody transfer. Features such as dots and squares of 1 and $2 \mathrm{~mm}$ in size, text and fine lines (down to $30 \mu \mathrm{m}$ nominal width) were reproduced effectively. Refrigerated samples of printed antibodies were found to function when tested one month after printing.

Figure 7. Printed anti-FITC serum visualized by binding anti-sheep-peroxidase then chemiluminescence - 10 seconds exposure - ink uses $2 \%$ anti-FITC serum and $2.5 \%$ high molecular weight PVA in carbonate buffer.

Patterning of nitrocellulose. When patterning the nitrocellulose coating using the IGT F1, printed features such as dots and fine lines were accurately produced, making it suitable for patterning antibody receptive areas for subsequent antibody printing (figure 8 shows printed $1 \mathrm{~mm}$ dots and squares, with dye to aid visualisation). The coating was also found to pattern effectively using the IGT G1. The low nitration (A-grade) nitrocellulose gave better results as it had a less volatile solvent blend, which prevented premature drying of the ink.

Figure 8. Nitrocellulose coating patterned using flexography onto acrylic coated polypropylene substrate (dye incorporated for visualization). 


\section{Discussion and conclusions}

An antibody ink was developed, using $2.5 \%$ high molecular weight PVA and carbonate-bicarbonate buffer, and was patterned onto flexible plastic film using flexographic printing. The formulation allowed the antibody to bind to the surface and remain accessible for binding with a secondary antibody. Nitrocellulose coatings were produced which improved binding of the antibodies. The interaction of antibodies with the nitro groups is encouraged by using an alkaline buffer (carbonate-bicarbonate) to increase the negative charge on the carbonyl groups in the protein [26]. This coating should be suitable for most polymer films and can be applied by conventional coating methods or discretely patterned using printing to increase the signal to noise ratio. Further benefits are improved quality of printed features, removal of the need for surfactant in the ink (which was found to reduce antibody binding), and reduced sensitivity of the print to changes in printing parameters. The narrow operational window of inkjet, a process more commonly used for biological printing, would not permit the use of formulations without a surfactant $[1,11]$. Furthermore, the use of a custom coating gives consistency to the surface and knowledge of its chemical composition. Commercial coatings employ a range of additives such as plasticizers and waxes which will affect surface interactions and hence antibody binding. There are also surface treatments such as corona discharge which, though useful for graphics printing quality, tend to give variation in wetting over the surface and changes over time, which will affect the deposition of antibodies. However, aggressive solvents in highly nitrated E-grade nitrocellulose formulations can dissolve many types of flexographic plate thus limiting the choice of plate materials. For lower nitration, or A-grade, nitrocellulose it was possible to substitute much of the acetate with less aggressive alcohols. This reduces photopolymer plate damage, making this grade more appropriate for patterning in flexographic printing; although it contains fewer nitro groups for binding. A-grade nitrocellulose is a common resin system for decorative flexographic inks. As an alternative, gravure printing uses an engraved metal cylinder as the image carrier; since there is no intermediate 
plate to damage, there are no restrictions on solvent selection. Both processes have proved effective for patterning the coating.

The range of polymer additives tested gave different printing and performance characteristics. High molecular weight PVA gave a good balance, by increasing viscosity without being required in excessive amounts, and allowing antibodies to bind and function effectively. The more soluble, low molecular weight polymers are not suitable for this application, where the antibodies should be tethered to the surface, but are useful for the conjugate release stage in lateral flow tests [27] where they aid the removal of antibodies from the surface.

Although the IGT F1 is similar to a full scale flexographic printer, it works on single rather than continuous prints and does not continually re-ink the anilox and plate. A full scale printing press can operate at higher speeds, on a reel-to-reel basis and with a substantially wider substrate, producing in excess of one square meter of antibody print per second.

The developments described in this article allow arrays of antibodies to be printed at speeds far in excess of those previously attainable for large scale production of immunoassays. Furthermore, this process could be easily adapted for printing of other biological materials, including but not limited to enzymes, DNA, proteins, aptamers and cells. The technology is also applicable for printing onto papers or membranes, which was confirmed by printing tests but requires higher anilox volumes and flexographic plate modification. Antibody prints were found to be stable after storage at $4^{\circ} \mathrm{C}$ for one month, however longer term storage or appropriate packing, to enhance lifetime, were not explored.

\section{Acknowledgements}


The authors acknowledge the financial support from the A4B CIRP Project HE 09 COL 1022 from the Welsh Assembly Government and are grateful to Innovia Films Ltd for providing polymer films and Ig-Innovations for providing both antibodies and technical advice.

\section{Reference:}

[1] Delaney Jr, J. T; Smith P. J; Schubert, U. S. Inkjet Printing of Proteins. Soft Matter. 2009, 5, $4866-4877$.

[2] Stewart, T. N., Pierson, B. E., Aggarwal, R. and Narayan, R. J. (2009), Piezoelectric Inkjet Printing of a Cross-Hatch Immunoassay on a Disposable Nylon Membrane. Biotechnology Journal. 2009, 4, 206-209. doi: 10.1002/biot.200800234.

[3] Hasenbank, M. S; Edwards, T; Fu, E; Garzon, R; Kosar, T. F; Look, M; Mashadi-Hossein, A; Yager, P. Demonstration of Multi-Analyte Patterning using Piezoelectric Inkjet Printing of Multiple Layers. Analytica Chimica Acta. 2008, 611, 80-88.

[4] Hossain, S. M. Z; Luckham, R. E; Smith, A. M; Lebert, J. M; Davies, L. M; Pelton, R. H; Filipe, C. D. M; Brennan, J. D. Development of a Bioactive Paper Sensor for Detection of Neurotoxins Using Piezoelectric Inkjet Printing of Sol-Gel-Derived Bioinks. Anal. Chem. 2009, 81, 5474-5483.

[5] Abe, K; Kotera, K; Suzuki, K; Citterio, D. Inkjet-Printed Paperfluidic Immuno-Chemical Sensing Device. Anal Bioanal Chem. 2010, 398, 885-893

[6] Austin, J; Holway, A. H. Contact Printing of Protein Microarrays. Methods in Molecular Biology. 2011, 785, 4, 379-394.

[7] Bernard, A; Delamarche, E; Schmid, H; Michel, B; Bosshard, H. R; Biebuyck, H. Printing Patterns of Proteins. Langmuir. 1998, 14, 9, 2225-2229.

[8] Delehanty, J. B. Printing Functional Protein Microarrays Using Piezoelectric Capillaries. Methods in Molecular Biology. 2004, 264, 135-143. 
[9] Kang, X; Li, Y, Fan, L, Lin, F, Wei, J; Zhu, X; Hu, Y; Li, J; Chang, G; Zhu, Q; Liu, H; Yang, Y. Development of an ELISA-Array for Simultaneous Detection of Five Encephalitis Viruses. Virology Journal. 2012, 9:56.

[10] Pelton, R. Bioactive Paper Provides a Low-Cost Platform for Diagnostics. Trends in Analytical Chemistry, 2009, 28, 8, 925-942

[11] Di Risio, S; Yan, N. Piezoelectric Ink-Jet Printing of Horseradish Peroxidase: Effect of Ink Viscosity Modifiers on Activity. Macromol. Rapid Commun. 2007, 28, 1934-1940.

[12] Product literature for BIODOT RR4510, BioDot, Inc., CA, USA. Available from www.biodot.com.

[13] Product literature for VersaArt ${ }^{\mathrm{TM}}$ RS-640, Roland DG (UK) Ltd., Cardiff, UK. Available from www.rolanddg.co.uk.

[14] Kipphan, H. Handbook of Print Media, Springer-Verlag. 2001, ISBN 3-540-67326-1.

[15] Leach, R. H; Pierce, R. J. The Printing Ink Manual, Fifth Edition, Kluwer Academic Publishers. 1993, ISBN 0-948905-81-6.

[16] Deganello, D; Cherry, J. A; Gethin, D. T; Claypole, T. C. Patterning of Micro-Scale Conductive Networks using Reel-to-Reel Flexographic Printing. Thin Solid Films. 2010, 518, 6113-6116.

[17] Wu P; Grainger, D. W. Comparison of Hydroxylated Print Additives on Antibody Microarray Performance. Journal of Proteome Research. 2006, 5, 2956-2965.

[18] Derham, B. K; Harding, J. J. The Effect of the Presence of Globular Proteins and Elongated Polymers on Enzyme Activity. Biochimica et Biophysica Acta. 2006, 1764, 1000-1006.

[19] Mahler, H-C; Senner, F; Maeder, K; Mueller, R. Surface Activity of a Monoclonal Antibody. J Pharm Sci. 2009, 98, 4525-4533.

[20] Biddlecome, J. G; Smith, G; Udin, S: Mulot, S: Spencer, D; Gee, C; Fish, B. C; Bracewell, D. G. Factors Influencing Antibody Stability at Solid-Liquid Interfaces in a High Shear Environment. Biotechnol. Prog. 2007, 23, 1218-1222. 
[21] Beer, H. H; Jallerat, E; Pflanz, K; Klewitz T, M. Qualification of Cellulose Nitrate Membranes for Lateral-Flow Assays. IVD Technology. 2002, 8, 35-42.

[22] Towbin, H; Staehelin, T; Gordon, J. Electrophoretic Transfer of Proteins from Polyacrylamide Gels to Nitrocellulose Sheets_-Procedure and Some Applications. Proc Natl Acad Sci USA. 1979, $76,4350-5354$.

[23] Millipore Corporation. Rapid Lateral Flow Test Strips: Considerations for Product Development. Lit. No. TB500EN00. Bedford, MA: 2002.

[24] Jones, K. D. Troubleshooting Protein Binding in Nitrocellulose Membranes. IVD Technology 1999, 5, 32-41.

[25] Pitt, A. M. The Nonspecific Protein Binding of Polymeric Microporous Membranes. J Parenteral Sci Tech. 1987, 41, 110-113.

[26] Peacock, D. G; Richardson J. F. (Ed). Chemical Engineering Volume 3, Third Edition, Pergamon. 1994, ISBN 0-08-041003-0.

[27] Wong, R. C; Tse, H. (Ed). Lateral Flow Immunoassay, Humana Press. 2009, ISBN: 978-158829-908-6 e-ISBN: 978-1-59745-240-3. DOI 10.1007/978-1-59745-240-3. 


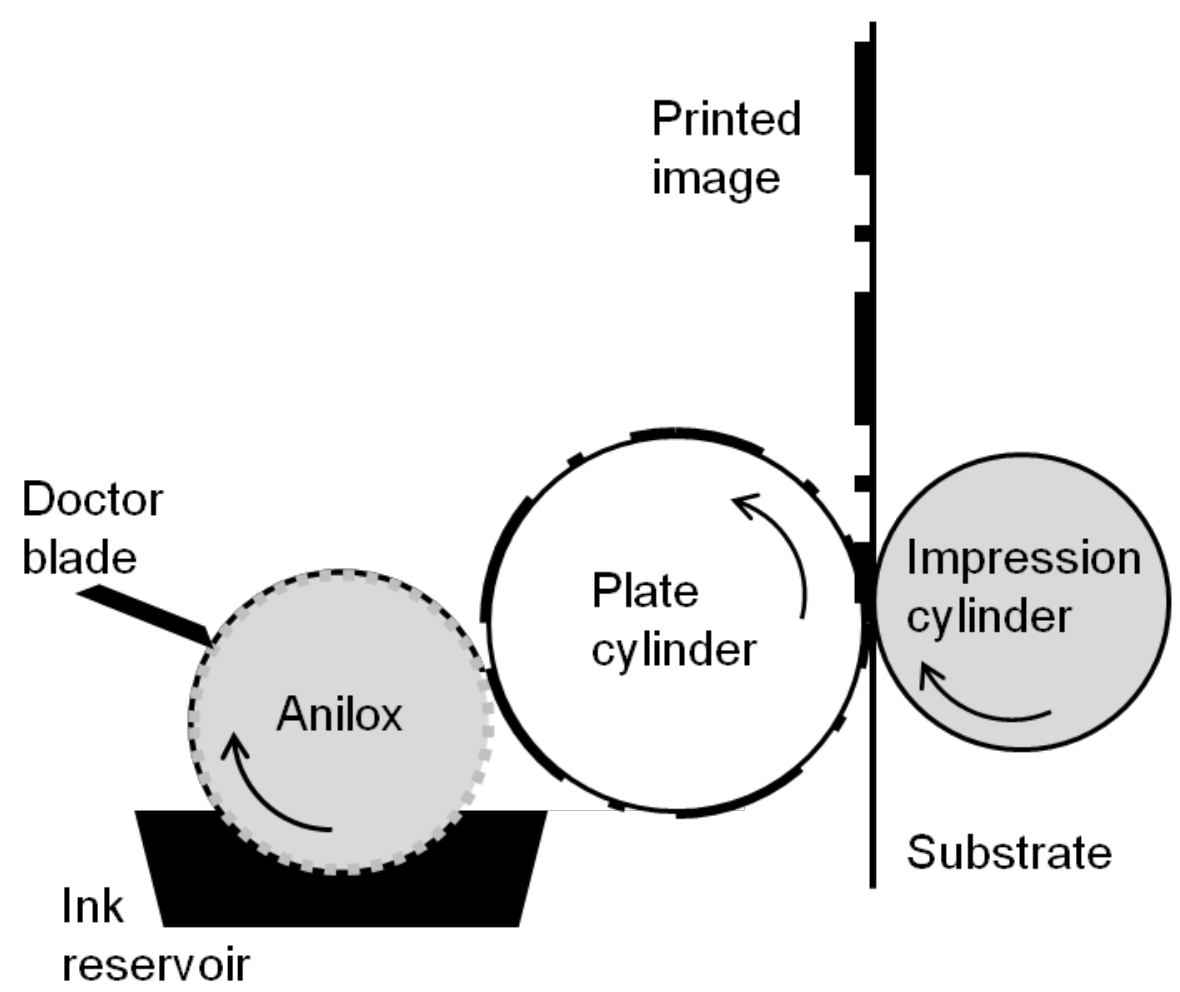

Figure 1. Schematic of the flexographic printing process.

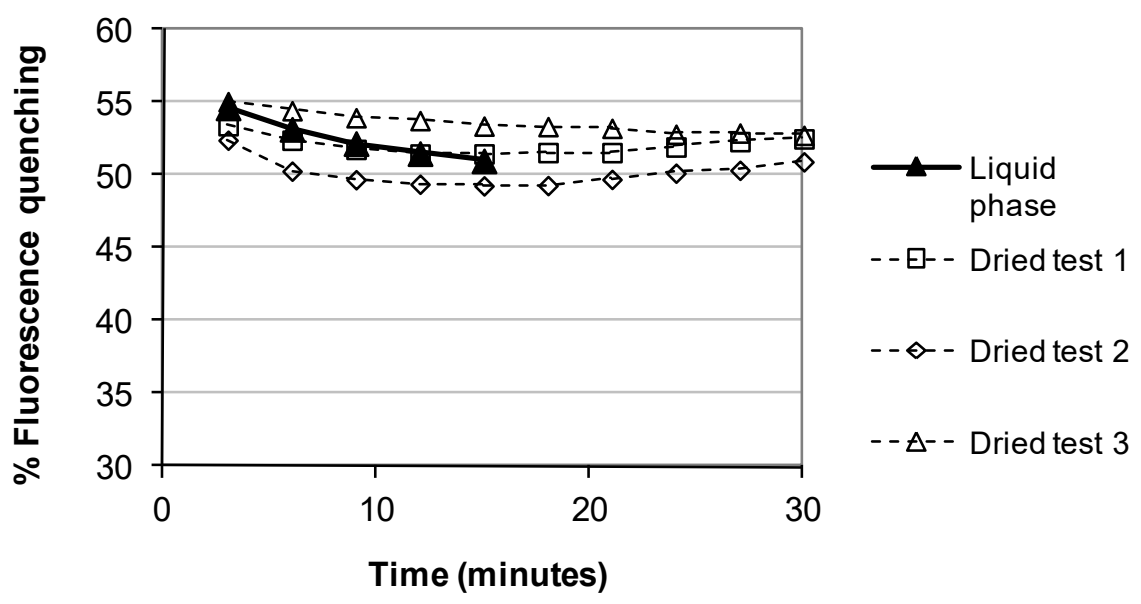

Figure 2. Fluorescence quenching for anti-FITC in PBS compared with rehydrated anti-FITC dried in PBS. 


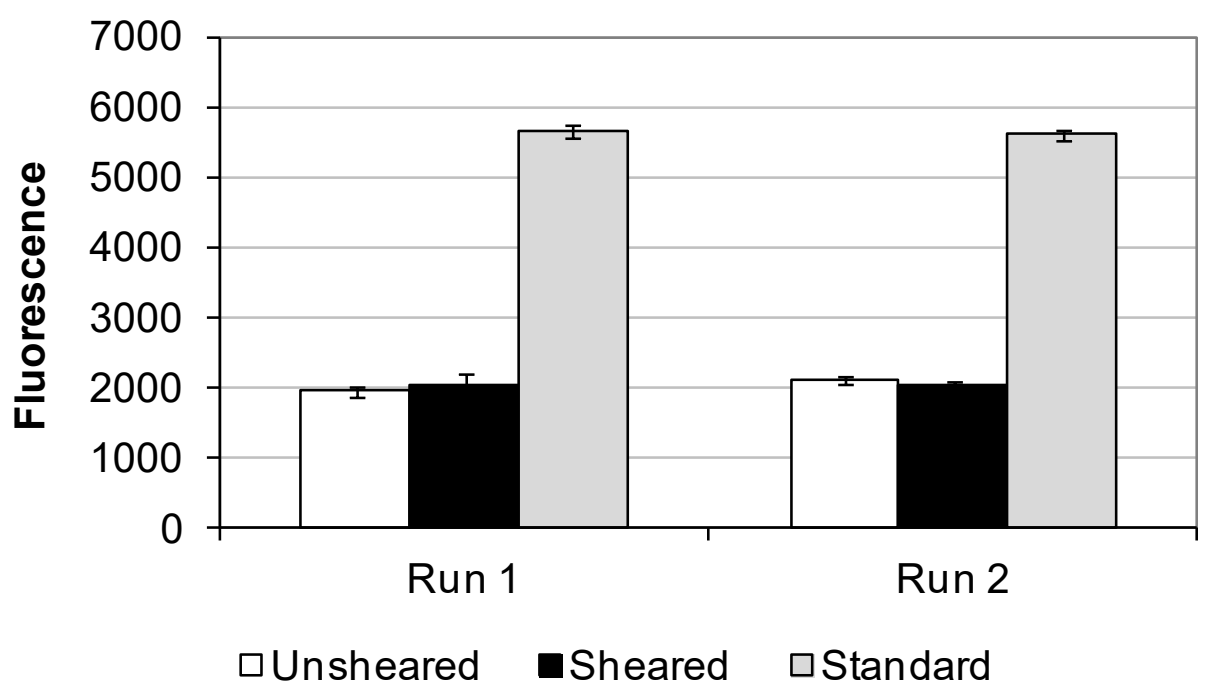

Figure 3. Fluorescence quenching for unsheared anti-FITC and anti-FITC sheared at $25,000 \mathrm{~s}^{-1}$ for 30 minutes $(0.025 \%$ antiserum with $10 \mathrm{nM}$ Fluorescein (error bars show standard deviation)).

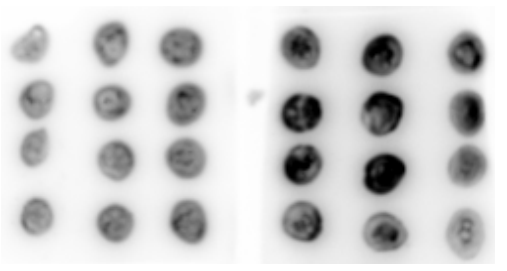

Figure 4. Anti-Sheep IgG (whole molecule)-Peroxidase, visualized by chemiluminescence, on acrylic (left) and nitrocellulose coated surfaces (right). Average intensity of the chemiluminescent signal for the droplets was 1.6 times higher on the nitrocellulose coating than the acrylic surface. Formulation contains $0.25 \%$ antibody and $2.5 \%$ high molecular weight PVA in carbonate buffer. 


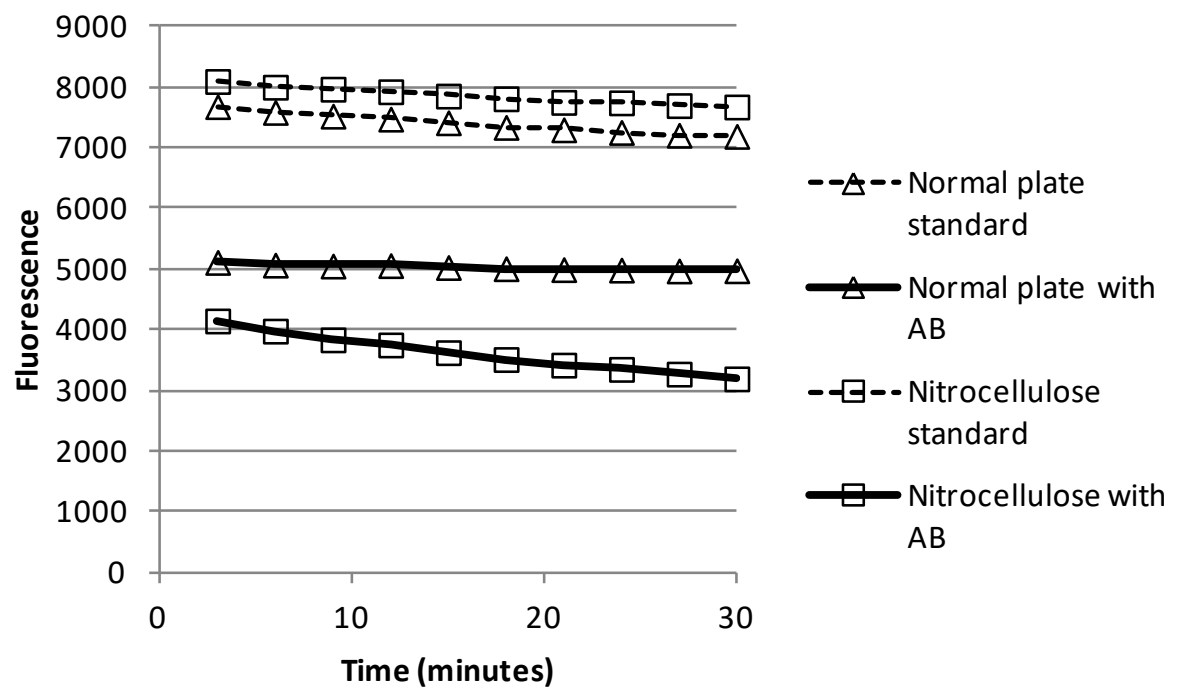

Figure 5. Comparison of dried then washed antibody deposit on normal polystyrene plate surface and nitrocellulose coated surface. Formulation contains 2\% anti-FITC serum and 2.5\% high molecular weight PVA in carbonate buffer. 


\section{print \\ dy print prine \\ ody print ty print \\ jody print ody prin? body print body print}

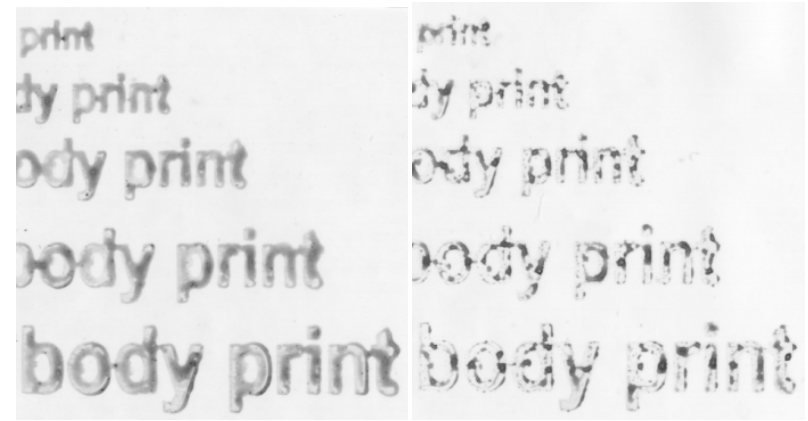

Figure 6. Printed dye on nitrocellulose (left) and acrylic (right) using $8 \mathrm{~mL} / \mathrm{m}^{2}$ anilox and $1 \mathrm{~m} / \mathrm{s}$ print speed (top) and $24 \mathrm{~mL} / \mathrm{m}^{2}$ anilox and $0.2 \mathrm{~m} / \mathrm{s}$ print speed (bottom) - ink uses $2.5 \%$ high molecular weight PVA in carbonate buffer.
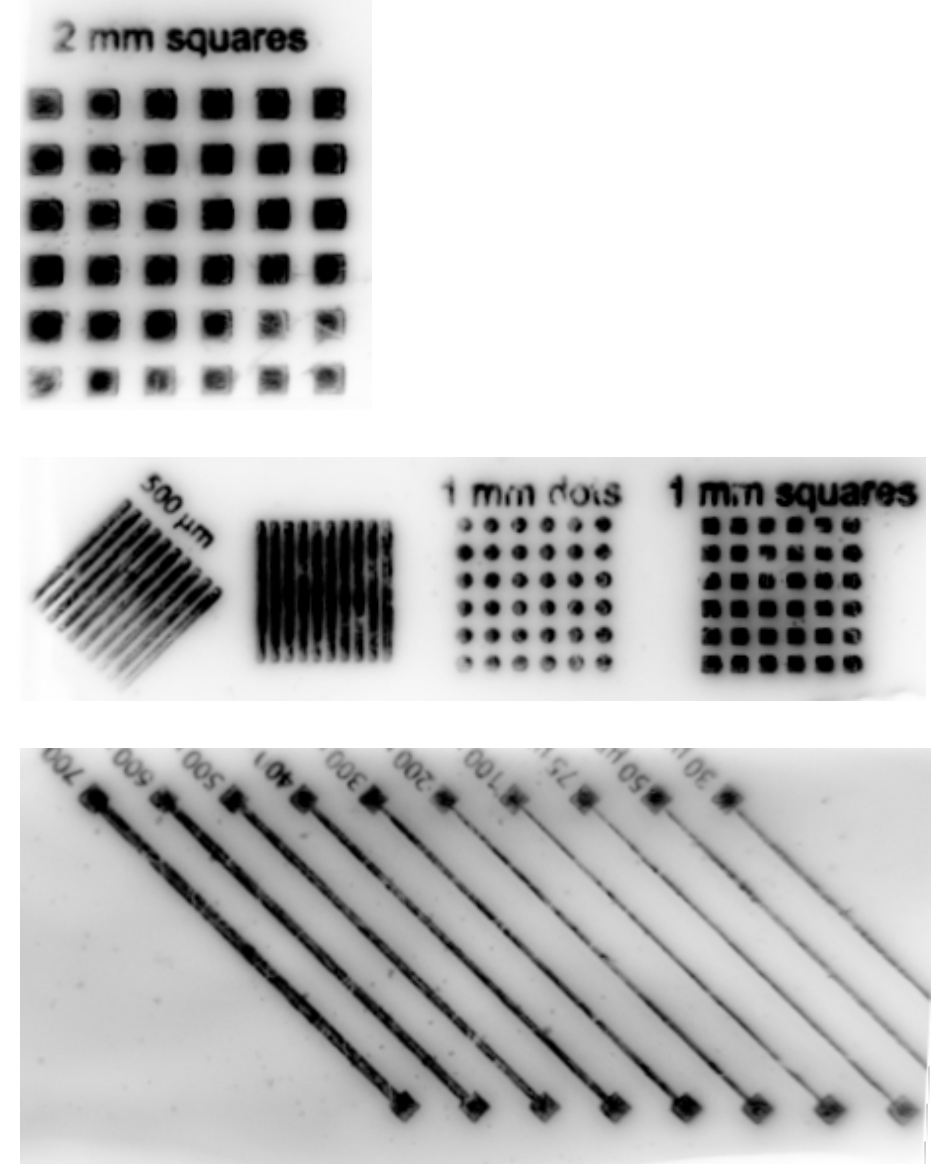
Figure 7. Printed anti-FITC serum visualized by binding anti-sheep-peroxidase then chemiluminescence - 10 seconds exposure - ink uses 2\% anti-FITC serum and 2.5\% high molecular weight PVA in carbonate buffer.

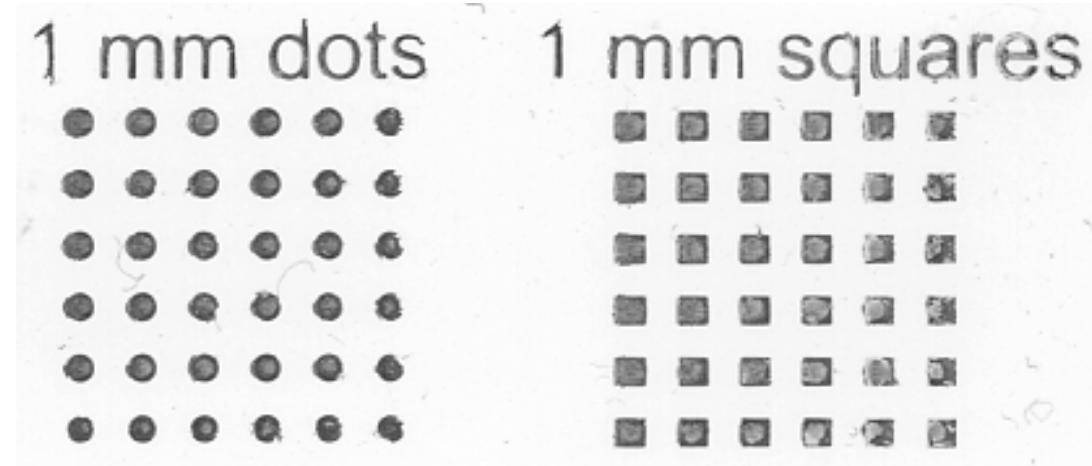

Figure 8. Nitrocellulose coating patterned using flexography onto acrylic coated polypropylene substrate (dye incorporated for visualization).

table of contents only 